\title{
On Homotopy of Walks and Spherical Maps in Homotopy Type Theory
}

\author{
Jonathan Prieto-Cubides \\ Department of Informatics \\ University of Bergen \\ Bergen, Norway \\ jonathan.cubides@uib.no
}

\begin{abstract}
We work with combinatorial maps to represent graph embeddings into surfaces up to isotopy. The surface in which the graph is embedded is left implicit in this approach. The constructions herein are proof-relevant and stated with a subset of the language of homotopy type theory.

This article presents a refinement of one characterisation of embeddings in the sphere, called spherical maps, of connected and directed multigraphs with discrete node sets. A combinatorial notion of homotopy for walks and the normal form of walks under a reduction relation is introduced. The first characterisation of spherical maps states that a graph can be embedded in the sphere if any pair of walks with the same endpoints are merely walk-homotopic. The refinement of this definition filters out any walk with inner cycles. As we prove in one of the lemmas, if a spherical map is given for a graph with a discrete node set, then any walk in the graph is merely walk-homotopic to a normal form.

The proof assistant Agda contributed to formalising the results recorded in this article.

CCS Concepts: • Theory of computation $\rightarrow$ Constructive mathematics; Type theory; - Mathematics of computing $\rightarrow$ Graphs and surfaces.
\end{abstract}

Keywords: graph maps, walk normal forms, HoTT, Agda

\section{ACM Reference Format:}

Jonathan Prieto-Cubides. 2022. On Homotopy of Walks and Spherical Maps in Homotopy Type Theory. In Proceedings of the 11th ACM SIGPLAN International Conference on Certified Programs and Proofs (CPP '22), Fanuary 17-18, 2022, Philadelphia, PA, USA. ACM, New York, NY, USA, 14 pages. https://doi.org/10.1145/3497775.3503671

Permission to make digital or hard copies of all or part of this work for personal or classroom use is granted without fee provided that copies are not made or distributed for profit or commercial advantage and that copies bear this notice and the full citation on the first page. Copyrights for components of this work owned by others than the author(s) must be honored. Abstracting with credit is permitted. To copy otherwise, or republish, to post on servers or to redistribute to lists, requires prior specific permission and/or a fee. Request permissions from permissions@acm.org. CPP '22, fanuary 17-18, 2022, Philadelphia, PA, USA

( $) 2022$ Copyright held by the owner/author(s). Publication rights licensed to ACM.

ACM ISBN 978-1-4503-9182-5/22/01...\$15.00

https://doi.org/10.1145/3497775.3503671

\section{Introduction}

This paper investigates the notion of homotopy of walks to study an equivalence between two definitions of embeddings in the sphere of connected and locally finite directed multigraphs. The constructions are proof-relevant and constructive, powered by homotopy type theory (HoTT) as the chosen mathematical foundation [7, 26].

The topological graph theory approach inspires our definition of a combinatorial notion of embedding/map in the sphere for graphs [23], referred to as spherical maps in this paper, see Definition 5.4. A graph map can be described by the graph itself and the circular ordering of the edges incident to each vertex [10, §3]. Using this description, a graph is understood to be embedded in the sphere if the walks with the same endpoints are walk-homotopic, similar to the topological concept of a connected closed and simply connected space. We propose a more pragmatic characterisation of spherical maps, using the fact that cycles/loops in the graph are walk-homotopic to a point in the sphere. To prove a map is spherical for a graph with a discrete node set, it is unnecessary to consider the infinite collection of walks. The set of walks without inner loops suffices, as we proved in Corollary 5.8.

To demonstrate our main results, we introduce a reduction relation and the notion of quasi-simple walks in Definitions 4.8 and 4.29, respectively. Using this reduction relation, as stated in Theorem 4.37, it is possible to define a normal form for walks and prove that every walk always has a normal form under certain conditions. Additionally, suppose a spherical map is given for a graph with a discrete node set. In this case, we provide a normalisation theorem to state that any walk is merely walk-homotopic to a normal form, see the details in Theorem 5.7.

Outline. The terminology and notation used throughout the paper is presented in Section 2. Readers familiar with HoTT may want to skip this section. The type of graphs discussed in this paper is defined in Section 3. In Section 4, we define the type of walks and the type of quasi-simple walks to introduce the normal form of a walk in Section 4.6. In Section 5 , a normalisation theorem for walks is given. Related work is reviewed in Section 6, and finally, conclusions are drawn and future work outlined in Section 7. 
Computer Formalisation. One advantage of using dependent type theories, as in this paper, is checking the correctness of the mathematical constructions using computer assistance. A proof assistant is a system with support to write such programs/proofs. The results in this document were formalised in the proof assistant Agda v(2.6.2), in a fully self-contained development, which does not depend on any library. The digital version of this document contains links to the Agda terms for some definitions, lemmas, and proofs. For example, we have made clickable the QED symbol ( $\square$ ) at the end of a proof.

In the implementation, the formalisation is type-checked using the flag wi thout-K for compatibility with HoTT [4]. Also, the flag exact-split was used to ensure that all clauses in a definition are definitional equalities. In our Agda library, to support this development, we required only a postulate for function extensionality and the corresponding postulates related to propositional truncation.

\section{Mathematical Foundation}

Homotopy type theory (HoTT) is an intensional Martin-Löf type theory (MLTT) [1,26] containing Voevodsky's Univalence axiom [27] and some higher inductive types, such as propositional truncation.

Revealed thanks to the formalisation, only a subset of HoTT is required for the results of this work. Precisely, we only need MLTT with universes, function extensionality and propositional truncation. However, since this work is part of a more ambitious project in which the whole theory is used, let us say that HoTT is our mathematical foundation for studying graph theory. This approach gives us, for example, the correct encoding of the equality between graphs, in the sense of the identity type, coinciding with the notion of graph isomorphism.

In HoTT, there is a natural correspondence between homotopy theory and the higher structure of the identity type of intensional MLTT. A space is a type where points are terms of their corresponding type, and paths from $a$ to $b$ are of the identity type between $a$ and $b$. By such a correspondence, one can, for example, study synthetic homotopy theory, as presented in the HoTT Book [26, §8].

An informal type theoretical notation derived from the HoTT book [26] and the formal system Agda [19] is used throughout the paper. Definitions are introduced by $(: \equiv)$ while judgmental equalities use (三). The identity type is denoted by (=). The universe is denoted by $\mathcal{U}$. The notation $A: \mathcal{U}$ indicates that $A$ is a type. To state that $a$ is of type $A$ we write $a: A$. The universe $\mathcal{U}$ is closed under the following type formers. The coproduct of two types, $A$ and $B$, is denoted by $A+B$. The corresponding data constructors are the functions inl $: A \rightarrow A+B$ and inr $: B \rightarrow A+B$. The dependent sum type ( $\sum$-type) is denoted by $\sum_{x: A} B(x)$. The dependent product type (П-type) is denoted by $\Pi_{x: A} B(x)$. The empty type and unit type are denoted by $\mathbb{O}$ and $\mathbb{1}$, respectively. The type $x \neq y$ denotes the function type $(x=y) \rightarrow \mathbb{O}$. Natural numbers are of type $\mathbb{N}$. $0: \mathbb{N}$. The successor of $n: \mathbb{N}$ is denoted by $S(n)$ or $n+1$. Given $n: \mathbb{N}$, the type with $n$ elements is denoted by $\llbracket n \rrbracket$ and is defined inductively by setting $\llbracket 0 \rrbracket: \equiv \mathbb{O}, \llbracket 1 \rrbracket: \equiv \mathbb{1}$ and $\llbracket n+1 \rrbracket: \equiv \llbracket n \rrbracket+\mathbb{1}$. To define some inductive types, we adopt a similar notation as in Agda, including the keyword data and the curly braces for implicit arguments, e.g. $\{a: A\}$ denotes $a$ is of type $A$, and it is an implicit variable. The type may be omitted in the former notation, as they can usually be inferred from the context.

We follow the HoTT Book, with slight changes in notation, for definitions such as embeddings, equivalence of types denoted by $(\simeq)$, propositional truncation of type $A$ denoted by $\|A\|$, and $n$-types, e.g. contractible types, propositions, and sets, with their corresponding predicate, isContr, isProp, and isSet.

Theorem 2.1 (Hedberg's theorem). A type A with decidable equality, i.e. $x=y$ or $x \neq y$ for all $x, y: A$, forms a set, and it is below referred to as discrete set.

It remains to define two fundamental notions towards studying the combinatorics of graphs, namely the type of finite sets and cyclic sets.

Definition 2.2. Given $X: \mathcal{U}$, let isfinite $(X): \mathcal{U}$ be given by

$$
\operatorname{isFinite}(X): \equiv \sum_{(n: \mathbb{N})}\|X \simeq \llbracket n \rrbracket\|
$$

The finiteness of a type $X$ is the existence of a bijection between $X$ and the type $\llbracket n \rrbracket$ for some $n: \mathbb{N}$. One can prove that Type (2.1) is a proposition. A type $X$ is called finite if isFinite $(X)$ holds. The corresponding natural number $n$ is referred as the cardinal number of $X$. Any property on $\llbracket n \rrbracket$, for example, "being a set" and "being discrete", can be transported to any finite type.

Lemma 2.3. Finite sets are closed under (co) products, type equivalences, $\Sigma$-types and $\Pi$-types.

For example, if $A$ is a finite set and $B: A \rightarrow \mathcal{U}$ is a type family such that for each $a: A$ the type $B(a)$ is a finite set, one can conclude that the type $\Pi_{x: A} B(x)$ is a finite set. The formal proof of Lemma 2.3 and other related lemmas can be found in the Coq-HoTT library [2]. For example, one of such lemmas, used to demonstrate Lemma 4.20, states that the cardinality of $X$ is less than or equal to the cardinality of $Y$ if there exists an embedding from $X$ to $Y$.

As the very first examples of finite sets, we have the empty type, unit type, decidable propositions and the family of types $\llbracket n \rrbracket$ for every $n: \mathbb{N}$. To prove the finiteness of other types, as in Theorem 4.22, we use Lemma 2.4, a direct consequence of Hedberg's theorem and finiteness of the empty and unit type. 
Lemma 2.4. If $A$ is discrete, then the identity type $x=y$ is a finite set for all $x, y: A$.

We now present a definition of cyclic types, used later to define the combinatorial characterisation of graphs embedded in a surface in Definition 5.1. Being cyclic for a type is a structure, not a property, given by preserving the structure of cyclic subgroups of permutations on $\llbracket n \rrbracket$. To endow a type with such a cyclic structure, let pred be the predecessor function of type $\llbracket n \rrbracket \rightarrow \llbracket n \rrbracket$, defined as the mapping, $0 \mapsto(n-1)$ and $(m+1) \mapsto m$ for $m<n$.

Definition 2.5. Given $A$ : $\mathcal{U}$, we define the type of cyclic structures on $A$, Cyclic $(A)$, as follows.

$\operatorname{Cyclic}(A): \equiv \sum_{(\varphi: A \rightarrow A)} \sum_{(n: \mathbb{N})}\left\|\sum_{(e: A \simeq \llbracket n \rrbracket)}(e \circ \varphi=\operatorname{pred} \circ e)\right\|$.

A cyclic structure is denoted by a tuple $\langle\varphi, n\rangle$ where $(\varphi, n, p)$ is of type Cyclic $(A)$. One may omit $n$ for brevity if no confusion arises. A type $A$ with a cyclic structure $\langle\varphi, n\rangle$ is referred as an $n$-cyclic type or simply as a cyclic set with $n$ elements.

\section{The Type of Graphs}

A graph is a term of the type in Definition 3.1. The corresponding data is a set of nodes and a set for each pair of nodes called edges.

Definition 3.1. A directed multigraph is of the following type.

$$
\text { Graph }: \equiv \sum_{(\mathrm{N}: \mathcal{U})} \sum_{(\mathrm{E}: \mathrm{N} \rightarrow \mathrm{N} \rightarrow \mathcal{U})} \operatorname{isSet}(\mathrm{N}) \times \prod_{(x, y: \mathrm{N})} \operatorname{isSet}(\mathrm{E}(x, y))
$$

Given a graph $G$, the set of nodes is denoted by $\mathrm{N}_{G}$. Given two nodes $x$ and $y$, the edges between them form a set denoted by $\mathrm{E}_{G}(x, y)$. If $e$ is an edge from $x$ to $y$, we denote by source $(e)$ the node $x$ and by $\operatorname{target}(e)$ the node $y$. A finite graph is a graph where the node set is a finite set as well as every family of sets $\mathrm{E}_{G}(x, y)$. One can prove that the type of graphs in Definition 3.1 forms a homotopy groupoid and is also a univalent category [26]. The proof of these facts and related lemmas will be omitted as it is not essential for our work here. The interested reader can check the formalisation in Agda for the respective proofs [21]. In the upcoming sections, unless stated otherwise, we will denote $G$ to be a graph, and $x, y$, and $z$ to be variables for nodes in $G$.

\section{Walks in a Graph}

The notion of a walk plays an essential role in graph theory. Many of the algorithms using graph data structures are based on this object. One may be interested in finding the "distance between two nodes" in a graph, the shortest walk, and several other variation problems related to walking in the graph.

Definition 4.1. A walk in $G$ from $x$ to $y$ is a sequence of connected edges that we construct using the following inductive data type:

$$
\begin{aligned}
\text { data } \mathrm{W}: & \mathrm{N}_{G} \rightarrow \mathrm{N}_{G} \rightarrow \mathcal{U} \\
\left\langle \_\right\rangle: & \left(x: \mathrm{N}_{G}\right) \rightarrow \mathrm{W}_{G}(x, x) \\
\left(\odot_{-}\right): & : \Pi\left\{x y z: \mathrm{N}_{G}\right\} \cdot\left(e: \mathrm{E}_{G}(x, y)\right) \\
& \rightarrow\left(w: \mathrm{W}_{G}(y, z)\right) \\
& \rightarrow \mathrm{W}_{G}(x, z)
\end{aligned}
$$

Let $w$ be a walk from $x$ to $y$, i.e. of type $\mathrm{W}_{G}(x, y)$. We will denote by $x$ the head of $w$ and by $y$ the end of $w$. If $w$ is $\langle x\rangle$ then we refer to $w$ as trivial or one-point walk. If $w$ is of the form $(e \odot\langle x\rangle)$, then $w$ is the one-edge walk $e$. Nontrivial walks are of the form $(e \odot w)$ and a loop is a walk with the same head and end.

\subsection{Structural Induction for Walks}

By structural induction or pattern matching on a walk, we will refer to the elimination principle of the inductive type in Definition 4.1. An induction principle allows us to define outgoing functions from a type to a type family. For instance, if we want to use the induction principle to inhabit a predicate on the type of walks, $P: \Pi\left\{x y: \mathrm{N}_{G}\right\} . \mathrm{W}_{G}(x, y) \rightarrow \mathcal{U}$, one can inhabit Type (4.1). Given a walk $w: \mathrm{W}_{G}(x, y)$, to construct a term of type $P(w)$, the base case must first be constructed, i.e. give a term of type $P(\langle x\rangle)$, for every $x: \mathrm{N}_{G}$. Subsequently, we must prove the case for composite walks, i.e. $P(e \odot w)$. To show this, $P(w)$ is assumed for any walk $w$, and we construct a term of type $P(e \odot w)$ from this assumption. Thus, one gets $P(w)$ for any walk $w$. Another induction principle for walks is stated in Theorem 4.5.

$$
\begin{aligned}
& \prod_{\left(x: \mathrm{N}_{G}\right)} P(\langle x\rangle) \\
\times & \prod_{\left(x, y, z: \mathrm{N}_{G}\right)} \prod_{\left(e: \mathrm{E}_{G}(x, y)\right)} \prod_{\left(x, y: \mathrm{W}_{G}(y, z)\right)} P(w) \rightarrow P(e \odot w) \\
\rightarrow & \prod_{\left(w: \mathrm{W}_{G}(x, y)\right)} P(w) .
\end{aligned}
$$

The composition, also called concatenation, of walks is an associative binary operation on walks defined by structural induction on its left argument. Given walks $p: \mathrm{W}_{G}(x, y)$ and $q: \mathrm{W}_{G}(y, z)$, we refer to their composition as the composite denoted by $p \cdot q$. The node $y$ is called the joint of the composition. The length of the walk $w$ is denoted by length $(w)$ and represents the number of edges used to construct $w$. A trivial walk has length zero, whilst a walk $(e \odot w)$ has one more length than $w$. We display a point to represent trivial walks and with a normal arrow to represent walks of positive length, as illustrated in Figure 1.

Lemma 4.2. The type of walks forms a set.

Proof. One can show that the type $\mathrm{W}(x, y)$ is equivalent to $\Sigma_{n: \mathbb{N}} \hat{W}(n, x, y)$ with $\hat{W}$ defined as follows.

$$
\hat{W}: \mathbb{N} \rightarrow \mathrm{N}_{G} \rightarrow \mathrm{N}_{G} \rightarrow \mathcal{U}
$$




$$
\begin{aligned}
& \hat{W}(0, x, y): \equiv(x=y), \\
& \hat{W}(S(n), x, y): \equiv \sum_{\left(k: N_{G}\right)} \mathrm{E}_{G}(x, k) \times \hat{W}(n, k, y) .
\end{aligned}
$$

It suffices to show that the type $\hat{W}(n, x, y)$ forms a set for $n: \mathbb{N}$ which will be proven by induction on $n$. If $n=0$, one obtains the proposition $x=y$ which is a set. Consequently, we must now show that the type in Equation (4.2c) is a set. By the graph definition, the base type $\mathrm{N}_{G}$ and $\mathrm{E}_{G}$ are both sets. Thus, one only requires that $\hat{W}(n, k, y)$ forms a set, which is precisely the induction hypothesis.

Although it is not included in the formalisation of this work, one can show that the type of walks forms a category. If Graph is the category of graphs using Definition 3.1 and $C$ is the category of small categories. There is a functor $R$ : Graph $\rightarrow C$ mapping every graph $G$ to its free precategory. The object set of $R(G)$ is $\mathrm{N}_{G}$, and the morphisms correspond to the collection of all possible walks in $G$. By Lemma 4.2, it follows that $R(G)$ is a small category. Let $L$ be the forgetful functor from $C$ to Graph. Then, $L$ is the left adjoint of $R$. The graph of walks $W(G)$ of $G$ is given by the endofunctor $W$ : Graph $\rightarrow$ Graph, the monad from the composite $L \circ R$.

\subsection{A Well-Founded Order for Walks}

Structural induction is a particular case of a more general induction principle to define recursive programs called wellfounded or Noetherian induction. Recall that for the structural induction principle, one must always guarantee that every argument in a recursive call in the program is strictly smaller than its arguments. However, there is no reason to believe this will always be the case.

In constructive mathematics, a binary relation $R$ on a set $A$ is well-founded if every element of $A$ is accessible. An element $a: A$ is accessible by $R$, if $b: A$ is accessible for every $b R a$ $[18,26, \S 10.3]$. Then, if $a$ has the property that there is no $b$ such that $b R a$, then $a$ is vacuously accessible. If $(\leq)$ represents the less or equal than relation on the natural numbers, then the number zero is vacuously accessible by $\leq$ on $\mathbb{N}$.

Let us define a well-founded order for walks in a graph by considering their lengths, from where the well-founded induction for walks follows, see Theorem 4.5.

Definition 4.3. Given $p, q: \mathrm{W}_{G}(x, y)$ for $x, y: \mathrm{N}_{G}$, the relation $(\preccurlyeq)$ states that $p \preccurlyeq q$ when length $(p) \leq$ length $(q)$.

Lemma 4.4. The relation $(\leqslant)$ on $\Sigma_{x, y}: \mathrm{N}_{G} \mathrm{~W}_{G}(x, y)$ is wellfounded.

Proof. It follows from the fact that the poset $(\mathbb{N}, \leq)$ is wellfounded.

We refer to the following lemma as the well-founded induction principle for walks induced by Definition 4.3.

Theorem 4.5. Suppose the following is given,
1. a predicate $P$ of type $\Sigma_{x, y}: \mathrm{N}_{G} \mathrm{~W}_{G}(x, y) \rightarrow \mathcal{U}$ such that,

2. given $(a, b, q)$ of type $\Sigma_{x, y}: \mathrm{N}_{G} \mathrm{~W}_{G}(x, y)$, if $P(p)$ for each walk $p: \mathrm{W}_{G}\left(x^{\prime}, y^{\prime}\right)$ with $x^{\prime}, y^{\prime}: \mathrm{N}_{G}$ and $p \leqslant q$, then $P(a, b, q)$.

Then, given any walk $w: \mathrm{W}_{G}(x, y)$ and $x, y: \mathrm{N}_{G}$, we have $P(x, y, w)$.

Remark 1. The induction principle stated in Theorem 4.5 using Lemma 4.4 is equivalent to performing induction on the length of the walk.

Theorems 4.37 and 5.7 define algorithms for which many of their recursive calls are on subwalks of the input walk. A subwalk of a walk $w$ is a contiguous subsequence of edges in $w$. Subwalks are not structurally smaller than their corresponding walk, unless one takes for example the subwalk $w$ or $e$ for the composite walk $(e \odot w)$. Excluding the previous case, to deal with other subwalk cases, we can use the well-founded induction principle given in Theorem 4.5.

\subsection{Quasi-Simple Walks}

In this subsection, we characterise walks with shapes as in Figure 1 and refer to such as quasi-simple walks in Definition 4.8.

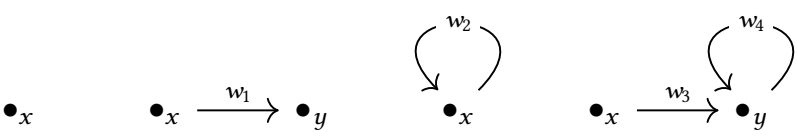

Figure 1. The arrows in the picture can represent edges or walks of a positive length. In the sense of Definition 4.8, a quasi-simple walk can only be one of these kinds: i) onepoint walk ii) path iii) loop without inner node repetitions, or iv) composite walk between a path and a quasi-simple walk of kind iii. The walks $w_{3}$ and $w_{4}$ only share the node $y$.

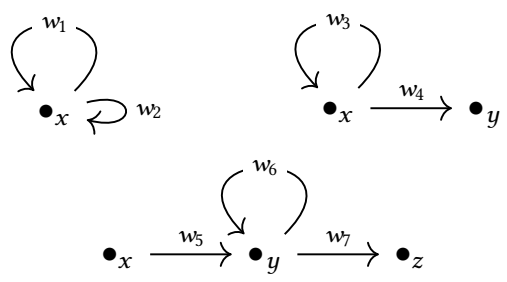

Figure 2. These are three examples of walks that are not quasi-simple in the sense of Definition 4.8. The walks $w_{1}$ and $w_{2}$ only share the node $x$, and the same happens with the walks $w_{3}$ and $w_{4}$. The walks $w_{5}, w_{6}$ and $w_{7}$ only share the node $y$. The walks $w_{i}$ for $i$ from 1 to 7 are nontrivial walks.

The notion of a quasi-simple walk will be used to introduce a reduction relation on the set of walks to remove their inner 
loops, see Definition 4.29. A related notion to the quasisimple walk definition is that of a path [5]. The usual graphtheoretical notion of a path is a walk with no repeated nodes. Here, quasi-simple walks are introduced since paths are not suitable in our description of graph maps in Section 5.2. There, the totality of walks is considered, which includes closed walks, also called loops. For graph maps in the sphere, we found out that the type of walks can be replaced by the type of quasi-simple walks under certain conditions. Quasiwalks are conveniently defined in a way that permits their end to appear at most twice in the walk.

To define quasi-simpleness for walks, we introduce a unconventional relation, denoted by $(x \in w)$, meaning that the node $x$ is in the walk $w$ and it is not the last, see Definition 4.6. $(x \in w)$ is a proposition, and decidable if the walks belong to graphs with discrete node set. Consequently, Lemma 4.13 shows that being quasi-simple is also a decidable proposition on the same kind of graphs. Quasi-simple walks play a relevant role in this work. They are required to give an alternative definition of graph maps in the sphere, as stated in Definition 5.5.

Definition 4.6. Let $x, y, z: \mathrm{N}_{G}$ and $w: \mathrm{W}_{G}(x, z)$. The relation $(\in)$ on a walk $w$ for a node $y$ is defined as the node $y$ that is not $z$ but belongs to $w$, i.e. whenever the type ( $y \in w)$ is inhabited.

1. $y \in\langle z\rangle: \equiv \mathbb{0}$.

2. $y \in(e \odot w): \equiv(y=\operatorname{source}(e))+(y \in w)$.

Lemma 4.7. If the node set of the graph $G$ is discrete, then the type $(x \in w)$ is decidable proposition for any node $x$ and walk $w$ in $G$.

Definition 4.8. Given $x, y: \mathrm{N}_{G}$, a walk in $G$ from $x$ to $y$ is quasi-simple if isQuasi $(w)$ holds.

$$
\operatorname{isQuasi}(w): \equiv \prod_{\left(z: N_{G}\right)} \operatorname{is} \operatorname{Prop}(z \in w) .
$$

Lemma 4.9. Being quasi-simple is a proposition.

Proof. It follows since is $\operatorname{Prop}(z \in w)$ is a proposition.

Thus, Definition 4.8 presents a quasi-simple walk as a path where the end could only be present at most twice. Examples of walks that are not quasi-simple are illustrated in Figure 2.

Lemma 4.10. Given $x, y, z: \mathrm{N}_{G}, e: \mathrm{E}_{G}(x, y)$ and a quasisimple walk $w: \mathrm{W}_{G}(y, z)$, if $x \notin w$ then the walk $(e \odot w)$ is quasi-simple.

Proof. Given a node $r$, we must show that $r \in(e \odot w)$ is a proposition. That is equivalent to showing that the type $(r=x)+(r \in w)$ is a proposition. The coproduct of mutually exclusive propositions is a proposition. Then, remember that $r=x$ is a given proposition and that the type $(r \in w)$ is also a proposition since the walk $w$ is quasi-simple by hypothesis. Thus, it remains to show that there is no term $(p, q)$ where $p:(r=x)$ and $q:(r \in w)$. A contradiction arises, since by hypothesis $x \notin w$ but from $\operatorname{tr}^{\lambda z \rightarrow z \in w}(p)(q): x \in w$.

Lemma 4.11. Given $x, y, z: \mathrm{N}_{G}, e: \operatorname{Edge}_{G}(x, y)$, and a walk $w: \mathrm{W}_{G}(y, z)$, if the walk $(e \odot w)$ is a quasi-simple walk then $w$ is also a quasi-simple walk.

Proof. Given any node $u: \mathrm{N}_{G}$ and two proofs $p, q: u \in w$, we must show that $p=q$. By definition, $\operatorname{inr}(p)$ and $\operatorname{inr}(q)$ are proofs that $u \in(e \odot w)$. Because $(e \odot w)$ is a quasi-simple walk, the equality $\operatorname{inr}(p)=\operatorname{inr}(q)$ holds. The constructor inr is an injective function, and one therefore obtains $p=q$ as required.

Corollary 4.12. Trivial and one-edge walks are quasi-simple walks.

Lemma 4.13. If the node set of the graph is discrete, then being quasi-simple for a walk is a decidable proposition.

Proof. Let $x, z: \mathrm{N}_{G}$ and $w: \mathrm{W}_{G}(x, z)$, we want to show that isQuasi $(w)$ is decidable. The proof is by induction on the structure of $w$.

1. If $w$ is trivial then, by Corollary 4.12 , the walk $w$ is quasi-simple.

2. If $w$ is the composite walk $\left(e \odot w^{\prime}\right)$ for $e: \mathrm{E}_{G}(x, y)$ and $w^{\prime}: W_{G}(y, z)$, we recursively ask whether the walk $w^{\prime}$ is quasi-simple or not.

a. If $w^{\prime}$ is not quasi-simple, then $w$ is not quasi-simple by the contrapositive of Lemma 4.11 .

b. If $w^{\prime}$ is quasi-simple, then we ask if $x \in w^{\prime}$. If so, then $w$ is not quasi-simple. Otherwise, that would contradict the quasi-simpleness definition, as the node $x$ would appear twice in $w$. Now, if $x \notin w^{\prime}$, one obtains that $w$ is quasi-simple by Lemma 4.10.

\subsection{A Finiteness Property}

The goal in this subsection is to prove that the collection of quasi-simple walks in a finite graph $G$ forms a finite set, as stated in Theorem 4.22. To show this, a proof on the finiteness of an equivalent type to Type (4.4) is given. To establish such equivalence, see Lemma 4.21, we first need to demonstrate some intermediate results as the following.

$$
\sum_{\left(w: W_{G}(x, y)\right)} \text { isQuasi }(w) .
$$

Lemma 4.14. Given any walk $w: \mathrm{W}_{G}(x, z)$ of length $n$, then

$$
\llbracket n \rrbracket \simeq \sum_{\left(y: N_{G}\right)}(y \in w) .
$$

Proof. By induction on the structure of $w$.

1. If the walk is trivial, the required equivalence follows from the type equivalence between $\mathbb{O}$ and $\Sigma_{z: \mathrm{N}_{G}} \mathbb{D}$. 
2. If the walk is $(e \odot w)$ for $e: \mathrm{E}_{G}(x, y)$ and $w: \mathrm{W}_{G}(y, z)$, the equivalence is established by the following calculation. Let $n$ be the length of $w$.

$$
\begin{aligned}
\sum_{\left(y: \mathrm{N}_{G}\right)}(y \in(e \odot w)) & \equiv \sum_{\left(y: \mathrm{N}_{G}\right)}(y=x)+(y \in w) \\
& \simeq \sum_{\left(y: \mathrm{N}_{G}\right)}(y=x)+\sum_{\left(y: \mathrm{N}_{G}\right)}(y \in w) \\
& \simeq \mathbb{1}+\sum_{\left(y: \mathrm{N}_{G}\right)}(y \in w) \\
& \simeq \mathbb{1}+\llbracket n \rrbracket \\
& \simeq \llbracket n+1 \rrbracket
\end{aligned}
$$

Equivalence (4.6a) is accomplished by Definition 4.6. $\sum$-type distributes coproducts as in Equivalence (4.6b). We can simplify in Equivalence (4.6c) because the type $\Sigma_{y: \mathrm{N}_{G}}(y=x)$ is contractible. Note that the inner path is fixed and it is then equivalent to the unit type. Equivalence (4.6d) is by the induction hypothesis applied to $w$. Equivalence (4.6e) is accomplished by the definition of $\llbracket n \rrbracket$ using the coproduct definition.

Lemma 4.15. Given $x, y, z: \mathrm{N}_{G}$, and $w: \mathrm{W}_{G}(x, y)$ the type $(z \in w)$ is a finite set if the node set of $G$ is discrete.

Proof. By induction on the structure of $w$ : in case the walk is trivial, the type in question is finite as it is equal to the empty type by definition. In the composite walk case, $z \in$ $(e \odot w)$, we must prove that the type $(z=x)+(z \in w)$ is finite. Note that the former is finite by Lemma 2.4. By the induction hypothesis: the type $z \in w$ is finite. The required conclusion then follows since finite sets are closed under coproducts.

We can now prove that for finite graphs there exists a finiteness property for the collection of all quasi-simple walks, derived from the finiteness of the set of quasi-simple walks of a fixed length $n$ for $n: \mathbb{N}$.

Definition 4.16. Given $x, y: \mathbb{N}_{G}$ and $n: \mathbb{N}$, the type qswalk collects all quasi-simple walks of a fixed length $n$.

$$
\text { qswalk }(n, x, y): \equiv \sum_{\left(w: \mathrm{W}_{G}(x, y)\right)} \operatorname{isQuasi}(w) \times(\text { length }(w)=n) \text {. }
$$

Lemma 4.17. Given a graph $G, n: \mathbb{N}$, and $x, z: \mathbb{N}_{G}$, the following equivalence holds.

$$
\text { qswalk }(S(n), x, z) \simeq \sum_{\left(y: \mathrm{N}_{G}\right)} \sum_{\left(e: \mathrm{E}_{G}(x, y)\right)} \sum_{(w: \text { qswalk }(n, y, z))}(x \notin w) .
$$

Proof. The back-and-forth functions are extensions of the functions derived from Lemmas 4.10 and 4.11 .

Lemma 4.18. Given a finite graph, $x, y: \mathbb{N}_{G}$ and $n: \mathbb{N}$, the type qswalk $(n, x, y)$ in Definition 4.16 is a finite set.
Proof. It suffices to show that the type qswalk $(n, x, y)$ is finite. The proof is by induction on $n$.

1. If $n=0$, the type defined by qswalk $(0, x, z)$ is equivalent to the identity type $x=y$, as the only walks of length zero are the trivial walks. Given that the node set is discrete, the path space $x=y$ is finite by Lemma 2.4.

2. Otherwise, given $x, z: \mathrm{N}_{G}$, we must prove that the type qswalk $(S(n), x, z)$ is finite, for $n: \mathbb{N}$, assuming that qswalk $(n, x, z)$ is finite. This is equivalent to showing that the equivalent type given by Equivalence (4.7) is finite. The required conclusion follows by Lemma 2.3 , as each type of the $\Sigma$-type in the right-hand side of the equivalence in Equivalence (4.7) is finite. The set $\mathrm{N}_{G}$ and the sets by $\mathrm{E}_{G}$ are each finite, as $G$ is a finite graph. The type qswalk $(n, y, z)$ is finite by induction hypothesis. Lastly, any decidable proposition is finite i.e. $\left(x \notin w^{\prime}\right)$ is finite.

Lemmas 4.20 and 4.21 prove the fact mentioned earlier on the node repetition condition in a quasi-simple walk. A node can only appear once in a quasi-simple walk, unless the node is the end of the walk. From now on, unless stated otherwise, we will refer to $n$ as the cardinality of $\mathrm{N}_{G}$ whenever the node set of the graph $G$ is finite. The number of nodes in any quasi-simple walk is bounded by $n+1$.

Lemma 4.19. Let $G$ be a finite graph. Then Type (4.8) is a finite set.

$$
\sum_{\left(x, y: \mathrm{N}_{G}\right)} \sum_{(m: \llbracket n+1 \rrbracket)} \mathrm{qswalk}(m, x, y) .
$$

Proof. The conclusion follows since finite sets are closed under $\sum$-types. $\mathrm{N}_{G}$ is finite since $G$ is a finite graph. $\llbracket n+1 \rrbracket$ is finite. The type qswalk $(m, x, y)$ is finite by Lemma 4.18 .

Lemma 4.20. Given a graph $G$ with finite node set of cardinality $n, x, y: \mathrm{N}_{G}$ and a quasi-simple walk $w: \mathrm{W}_{G}(x, y)$ of length $m$, then it holds that $m \leq n$.

Proof. It suffices to generate an embedding between the finite set $\llbracket m \rrbracket$ and the finite node set in $G$. Such an embedding is the projection function $\pi_{1}: \Sigma_{x: \mathrm{N}_{G}}(x \in w) \rightarrow \mathrm{N}_{G}$. Recall that the domain of the function $\pi_{1}$ is equivalent to $\llbracket m \rrbracket$ by Lemma 4.14.

Now, even when the type of walks forms an infinite set, thanks to Lemma 4.20 and Theorem 4.22 , we will be able to prove that for any nodes $x$ and $y$, the collection of quasisimple walks from $x$ to $y$ forms a finite set as long as the graph is finite.

Lemma 4.21. Given a graph $G$ with finite node set of cardinality $n$ and $x, y: \mathrm{N}_{G}$, the following equivalence holds.

$$
\sum_{\left(w: \mathrm{W}_{G}(x, y)\right)} \operatorname{isQuasi}(w) \simeq \sum_{(m: \llbracket n+1 \rrbracket)} \operatorname{qswalk}(m, x, y) .
$$




\section{Proof. Apply Lemma 4.20.}

It is not immediately clear that quasi-simple walks forms a finite set, even when the graph is finite. A quasi-simple walk can contain a loop at its terminal node. One might think there are infinitely many walks if each walk loops at its terminal nodes. However, it is by constraining walks to be quasi-simple that we obtain the finiteness property.

Theorem 4.22. The quasi-simple walks of a finite graph $G$ forms a finite set, i.e. Type (4.10) is inhabited.

$$
\operatorname{isFinite}\left(\sum_{\left(x, y: \mathrm{N}_{G}\right)} \sum_{(w: W(x, y))} \operatorname{isQuasi(}(w)\right) .
$$

Proof. The conclusion clearly follows from Lemmas 4.19 and 4.21, since finite sets are closed under type equivalences and $\Sigma$-types by Lemma 2.3 .

\subsection{Walk Splitting}

In this subsection, a function to split/divide a walk $w$ from $x$ to $z$ into subwalks, $w_{1}$ and $w_{2}$, is given. Such a division of $w$, of type Type (4.11), is handy e.g. for proving statements where the induction is not on the structure but on the length of the walk.

$$
\sum_{\left(y: \mathrm{N}_{G}\right)} \sum_{\left(w_{1}: \mathrm{W}_{G}(x, y)\right)} \sum_{\left(w_{2}: \mathrm{W}_{G}(y, z)\right)}\left(w=w_{1} \cdot w_{2}\right) .
$$

Let $x, y, z$ be variables for nodes in $G$ and let $w$ be a walk from $x$ to $z$, unless stated otherwise. We refer to the walk $w_{1}$ in Type (4.11) as a prefix of $w$ and $w_{2}$ as the corresponding suffix given $w_{1}$.

Definition 4.23. Given two walks $p$ and $q$ with the same head, one says that $p$ is a prefix of $q$ if the type $\operatorname{Prefix}(p, q)$ is inhabited.

$$
\begin{aligned}
\text { data Prefix }: & \Pi\{x, y, z\} . \mathrm{W}_{G}(x, y) \rightarrow \mathrm{W}_{G}(x, z) \rightarrow \mathcal{U} \\
\text { head }: & \Pi\{x y\} . \Pi\left\{w: \mathrm{W}_{G}(x, y)\right\} . \operatorname{Prefix}(\langle x\rangle, w) \\
\text { by-edge }: & \Pi\{x y z k\} . \Pi\left\{e: \mathrm{E}_{G}(x, y)\right\} \\
& \Pi\left\{p: \mathrm{W}_{G}(y, z)\right\} . \Pi\left\{q: \mathrm{W}_{G}(y, k)\right\} \\
& \rightarrow \operatorname{Prefix}(p, q) \rightarrow \operatorname{Prefix}(e \odot p, e \odot q)
\end{aligned}
$$

Lemma 4.24. Given a prefix $w_{1}$ for a walk $w$, we can prove that there is a term of type Type (4.12) named suffix $\left(w_{1}, w, t\right)$, referring to as the suffix of $w$ given $w_{1}$, where $t: w=w_{1} \cdot w_{2}$.

$$
\sum_{\left(w_{2}: \mathrm{W}_{G}(y, z)\right)}\left(w=w_{1} \cdot w_{2}\right) .
$$

Proof. For brevity, we skip the trivial cases for $w_{1}$ and $w$. The remaining cases are proved by induction; first, on $w_{1}$, and secondly on $w$. The resulting nontrivial case occurs when $w_{1}=e \odot p, w=e \odot q$ and $t: \operatorname{Prefix}(p, q)$ for two walks $p$ and $q$. By the induction hypothesis applied to $p, q$, and $t$, the term $\operatorname{suffix}(p, q, t)$ is obtained, from which one gets the suffix walk $w_{2}$ along with a proof $i: q=p \cdot w_{2}$. Thus, the required term is the pair $\left(w_{2}, \operatorname{ap}(e \odot-, i)\right)$.

We now encode the case where the walk $w$ is divided at the first occurrence of the node $y$, using the type family SplitAt $(w, y)$ defined in Definition 4.25 . The corresponding method to inhabit the type $\operatorname{Split} \operatorname{At}(w, y)$ is the function given in Lemma 4.26, assuming the node set in the graph is discrete. This walk splitting encoding is implicitly used in several parts of the proof of Theorem 5.7.

Definition 4.25. The type $\operatorname{SplitAt}(w, y)$ is the inductive type defined as:

$$
\begin{aligned}
\text { data SplitAt } & \{x z\}\left(w: \mathrm{W}_{G}(x, z)\right)\left(y: \mathrm{N}_{G}\right): \mathcal{U} \\
\text { nothing }: \Pi\{x y\} . \Pi\left\{w: \mathrm{W}_{G}(x, y)\right\} & \rightarrow(y \notin w) \\
& \rightarrow \operatorname{SplitAt}(w, y) \\
\text { just }: & \Pi\{x y\} \cdot \Pi\left\{w: \mathrm{W}_{G}(x, y)\right\} \\
\rightarrow & \left(p: \mathrm{W}_{G}(x, y)\right) \\
\rightarrow & \text { Prefix }(p, w) \rightarrow(y \notin p) \\
\rightarrow & \text { SplitAt }(w, y)
\end{aligned}
$$

Lemma 4.26. The type SplitAt $(w, y)$ is inhabited if the node set of the graph is discrete.

Proof. By induction on the structure of the walk.

1. If the walk is trivial, then the required term is nothingid, as by definition, $y \notin \mathbb{O}$.

2. If the walk is the composite $(e \odot w)$ with $e: \mathrm{E}_{G}\left(x, y^{\prime}\right)$ and $w: \mathrm{W}_{G}\left(y^{\prime}, z\right)$, we ask whether $y$ is equal to $x$ or not.

a. If $y=x$ then the required term is just $(\langle y\rangle$, head, id).

b. If $y \neq x$ then by the induction hypothesis on $w$ and $y$, the following cases need to be considered.

i. If the case is nothing, then there is enough evidence that $y \notin w$ and we use for the required term the nothing constructor.

ii. Otherwise, there is a prefix $w_{1}$ for $w$ and a proof $r: y \notin w_{1}$. Using $r$ and the fact $x \neq y$, we can construct $r^{\prime}: y \notin\left(e \odot w_{1}\right)$. Then, the term that we are looking for is just $\left(e \odot w_{1}\right.$, by-edge $\left.(p), r^{\prime}\right)$ of type SplitAt $(e \odot w, y)$, as required in the conclusion.

\subsection{Normal Forms for Walks}

In this subsection, a reduction relation in Definition 4.29 is established on the set of walks of equal endpoints. Some cases considered by such a relation are illustrated in Figure 3. This relation provides a way to remove loops from walks in a graph with a discrete set of nodes. The notion of normal form for walks presented in this work is based on the loop reduction relation in Definition 4.33 . 


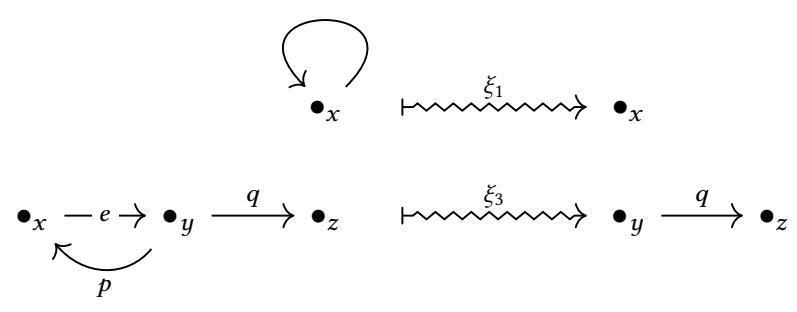

Figure 3. The rules $\xi_{1}$ and $\xi_{3}$ of the loop-reduction relation in Type (4.13).

The following definitions establish a few type families to encode walks of a certain basic structure-for example, nontrivial walks and loops-necessary for the formalisation.

Definition 4.27. Let $x, y: \mathrm{N}_{G}$ and $w: \mathrm{W}_{G}(x, y)$.

1. The walk $w$ is a loop whenever the head is equal to the end, i.e. $\operatorname{Loop}(w)$.

$$
\begin{aligned}
\text { data Loop }: & \Pi\{x, y\} . \mathrm{W}_{G}(x, y) \rightarrow \mathcal{U} \\
\text { is-loop : } & \Pi\{x y\} . \Pi\left\{w: \mathrm{W}_{G}(x, y)\right\} \\
\rightarrow & x=y \rightarrow \operatorname{Loop}(w)
\end{aligned}
$$

2. The walk $w$ is trivial if its length is zero, i.e. Trivial $(w)$.

$$
\begin{aligned}
& \text { data Trivial }: \Pi\{x, y\} \cdot \mathrm{W}_{G}(x, y) \rightarrow \mathcal{U} \\
& \text { is-trivial }: \Pi\{x y\} . \Pi\left\{w: \mathrm{W}_{G}(x, y)\right\} \\
& \rightarrow \text { length }(w)=0 \rightarrow \operatorname{Trivial}(w)
\end{aligned}
$$

3. A walk $w$ is not trivial, if it has one edge at least, i.e. $\operatorname{NonTrivial}(w)$.

data NonTrivial : $\Pi\{x, y\} . \mathrm{W}_{G}(x, y) \rightarrow \mathcal{U}$

$$
\text { has-edge } \begin{aligned}
: & \Pi\{x y z\} . \Pi\left\{w: \mathrm{W}_{G}(y, z)\right\} \\
& \rightarrow\left(e: \mathrm{E}_{G}(x, y)\right) \rightarrow \operatorname{NonTrivial}(e \odot w) .
\end{aligned}
$$

4. A walk $w$ does not reduce if $\operatorname{NoReduce}(w)$.

data NoReduce : $\Pi\{x, y\} . \mathrm{W}_{G}(x, y) \rightarrow \mathcal{U}$

$$
\begin{aligned}
\text { is-dot }: & \Pi\{x\} . \text { NoReduce }(\langle x\rangle) \\
\text { is-edge }: & \Pi\{x y\} . \Pi\left\{e: \mathrm{E}_{G}(x, y)\right\} \\
& \rightarrow(x \neq y) \rightarrow \operatorname{NoReduce}(e \odot\langle y\rangle)
\end{aligned}
$$

5. A walk $w$ is not a trivial loop if $\operatorname{NonTrivialLoop}(w)$.

$$
\begin{aligned}
& \text { data NonTrivialLoop }: \Pi\{x, y\} . \mathrm{W}_{G}(x, y) \rightarrow \mathcal{U} \\
& \text { is-loop }: \Pi\{x y z\} .\left\{e: \mathrm{E}_{G}(x, y)\right\} \\
& \rightarrow(p: x=z) \rightarrow\left(w: \mathrm{W}_{G}\right) \\
& \rightarrow \text { NonTrivialLoop }(e \odot w)
\end{aligned}
$$

Lemma 4.28. Given $x, y: \mathrm{N}_{G}$ and $u: \mathrm{W}_{G}(x, y)$, the following claims hold.

1. If $x \neq y$ then NonTrivial $(u)$.

2. If NonTrivial $(u)$ then $x \in u$.

3. Given $z: \mathrm{N}_{G}$, if $\operatorname{NonTrivial}(u)$ and $v: \mathrm{W}_{G}(y, z)$ then $\operatorname{NonTrivial}(u \cdot v)$.
Remember that a reduction relation $R$ on a set $M$ is an irreflexive binary relation on $M$. If $R$ is a reduction relation, we use $x R y$ to refer to the pair $(x, y)$ in $R$. If $x R y$ then one says that $x$ reduces to $y$ or simply $x$ reduces.

Definition 4.29. The loop-reduction relation $(\leadsto)$ on walks is Type (4.13).

$$
\begin{aligned}
\operatorname{data}(\rightsquigarrow) & : \Pi\left\{x, y: \mathrm{N}_{G}\right\} \cdot \mathrm{W}_{G}(x, y) \rightarrow \mathrm{W}_{G}(x, y) \rightarrow \mathcal{U} \\
\xi_{1} & : \Pi\{x y\} \cdot\left(p: \mathrm{W}_{G}(x, y)\right)\left(q: \mathrm{W}_{G}(x, y)\right) \\
& \rightarrow \operatorname{NonTrivialLoop}(p) \rightarrow \operatorname{Trivial}(q) \\
& \rightarrow p \rightsquigarrow q \\
\xi_{2} & : \Pi\{x y z\} \cdot\left(e: \mathrm{E}_{G}(x, y)\right)\left(p, q: \mathrm{W}_{G}(y, z)\right) \\
& \rightarrow \neg \operatorname{Loop}(e \odot p) \rightarrow x \neq y \\
& \rightarrow(p \rightsquigarrow q) \rightarrow(e \odot p) \rightsquigarrow(e \odot q) \\
\xi_{3} & : \Pi\{x y z\} \cdot\left(e: \mathrm{E}_{G}(x, y)\right)\left(p: \mathrm{W}_{G}(y, x)\right) \\
& \rightarrow\left(q: \mathrm{W}_{G}(x, z)\right) \\
& \rightarrow \neg \operatorname{Loop}((e \odot p) \cdot q) \rightarrow \operatorname{Loop}(e \odot p) \\
& \rightarrow \operatorname{NonTrivial}(q) \\
& \rightarrow\left(w: \mathrm{W}_{G}(x, z)\right) \rightarrow w=(e \odot p) \cdot q \\
& \rightarrow w \rightsquigarrow q
\end{aligned}
$$

The following provides hints to the intuition behind each of the data constructors above.

1. The rule $\xi_{1}$ is "a nontrivial loop reduces to the trivial walk of its endpoint".

2. The rule $\xi_{2}$ is "the relation $(\rightsquigarrow)$ is right compatible with edge concatenation".

3. The rule $\xi_{3}$ is "the relation $(\backsim)$ removes right attached loops".

Remark 2. The data constructors in Type (4.13) follow a design principle to avoid certain unification problems occurring in dependently type programs [12, 17].

Definition 4.30. The relation $\left(\varkappa^{*}\right)$ is the reflexive and transitive closure of the relation $(\leadsto)$ in Definition 4.29.

Lemma 4.31. Given $x, y: \mathrm{N}_{G}$ and $p, q: \mathrm{W}_{G}(x, y)$, the following claims hold:

1. If $x \in q$ and $p \leadsto * q$ then $x \in p$.

2. If $p \leadsto q$ then length $(q)<\operatorname{length}(p)$.

One can prove that our reduction relation in Definition 4.29 satisfies the progress property, similarly as proved for simplytyped lambda calculus in Agda [12, §2]. The evidence that a walk reduces is encoded using the following predicate.

Definition 4.32. Given a walk $p: \mathrm{W}_{G}(x, y)$,

$$
\operatorname{Reduce}(p): \equiv \sum_{\left(q: \mathrm{W}_{G}(x, y)\right)}(p \rightsquigarrow q) \text {. }
$$

The predicate Normal defined in Definition 4.33 is the evidence that a walk is a quasi-simple walk that can no longer reduce. 
Definition 4.33. Given a walk $p$, one states that $p$ is in normal form if $\operatorname{Normal}(p)$. If $p \rightsquigarrow q$ and $q$ is in normal form, we refer to $q$ as the normal formal of $p$.

$$
\operatorname{Normal}(p): \equiv \operatorname{isQuasi}(p) \times \neg \operatorname{Reduce}(p) \text {. }
$$

Lemma 4.34. Being in normal form for a walk is a proposition.

Proof. It follows from Lemmas 2.3 and 4.13 .

Example 4.35. The very basic normal forms for walks are the trivial ones and the one-edge walks with different endpoints. Given a walk $w$ and a term of $\operatorname{NoReduce}(w)$, one can easily show that the walk $w$ is in normal form.

Definition 4.36. Given nodes $x$ and $y$ in a graph $G$, we encode the fact a walk can reduce or not by using the inductive data type Progress.

$$
\begin{gathered}
\text { data Progress }\{x y\}\left(p: \mathrm{W}_{G}(x, y)\right): \mathcal{U} \\
\text { step : Reduce }(p) \rightarrow \operatorname{Progress}(p) \\
\text { done : } \operatorname{Normal}(p) \rightarrow \operatorname{Progress}(p)
\end{gathered}
$$

Theorem 4.37. Given a graph $G$ with a discrete node set, there exists a reduction for each walk to one of its normal forms, i.e. Type (4.14) is inhabited for all $w: \mathrm{W}_{G}(x, y)$.

$$
\sum_{\left(v: W_{G}(x, z)\right)}(w \rightsquigarrow * v) \times \operatorname{Normal}(v) .
$$

Remark 3. The reduction relation $(\leadsto)$ has the termination property. There is no infinite sequence of walks reducing, since the length of each walk in a chain like $w_{1} \rightsquigarrow w_{2} \rightsquigarrow w_{3} \rightsquigarrow \cdots$, decreases at each reduction step. See also Lemma 4.4.

Corollary 4.38. Given a graph $G$ with a discrete node set, and a walk $w$ of type $\mathrm{W}_{G}(x, y)$ for two $x, y: \mathrm{N}_{G}$, the following claims hold.

1. The type Reduce $(w)$ is decidable.

2. The proposition $\operatorname{Normal}(w)$ is decidable.

3. The walk $w$ progresses in the sense of Definition 4.36.

For simplicity, the proofs of Theorem 4.37 and Corollary 4.38 are omitted. Neither of them requires the law of excluded middle. However, if we want to construct the normal form for a walk, the node set of the graph has to be discrete. In the case of Theorem 4.37, its proof can use the same reasoning given for the proof of Theorem 5.7.

\section{The Notion of Walk Homotopy}

This section introduces the notion of homotopy for walks denoted by $\left(\sim_{\mathcal{M}}\right)$. We define such a relation in Definition 5.2 as a congruence relation on the category induced by the endofunctor $(\mathrm{W})$ on the corresponding graph. Because homotopy for walks depends on the surface in which the graph is embedded, it is necessary to first define an embedding of graphs in a surface.

A map/embedding of a graph is a cellular decomposition of the surface where the graph is embedded. This topological definition also requires defining what a surface is. To avoid this, we consider instead a combinatorial approach in Definition 5.1 based on the work by Edmonds and Tutte [24, 25]. A more complete description of graph maps can be found in $[10, \S 3]$.

Given a graph $G$, the graph formed by taking the same node set of $G$ and the edge set as the type $\mathrm{E}_{G}(x, y)+\mathrm{E}_{G}(y, x)$ for $x, y: \mathrm{N}_{G}$ is denoted by $U(G)$ and referred as the symmetrisation of $G$.

Definition 5.1. A map for a graph $G$ of type $\operatorname{Map}(G)$ is a local rotation system at each node in $U(G)$.

$$
\operatorname{Map}(G): \equiv \prod_{\left(x: \mathrm{N}_{G}\right)} \text { Cyclic }\left(\sum_{\left(y: \mathrm{N}_{G}\right)} \mathrm{E}_{U(G)}(x, y)\right) \text {. }
$$

Given a map $\mathcal{M}$, the faces of $\mathcal{M}$ are the regions obtained by the cellular decomposition of the corresponding surface by $\mathcal{M}$. We omit the formal type of faces herein, so as not to distract the reader from the goals of this paper. The type of faces requires proper attention [23]. Put briefly, a face is a cyclic walk in the embedded graph without repeating nodes and without edges inside [10]. The corresponding data of a face is a cyclic subgraph $A$ in $U(G)$ and a function $f: A \rightarrow N_{G}$ that picks nodes in $A$. Consequently, for each face $\mathcal{F}$ given by $\langle A, f\rangle$, there are at least two quasi-simple walks in $U(G)$ associated with $\mathcal{F}$ for every node-pair. Given $x, y: \mathrm{N}_{G}$, the corresponding walks given by $\mathcal{F}$ are, namely, the clockwise and counter-clockwise closed walks in $U(G)$, denoted by $\operatorname{cw}_{A}(x, y)$ and $\operatorname{ccw}_{A}(x, y)$, respectively. If the endpoints are equal, the trivial walk $\langle x\rangle$ must also be considered.

\subsection{Homotopy of Walks}

Definition 5.2. Let $w_{1}, w_{2}$ be two walks from $x$ to $y$ in $U(G)$. The expression $w_{1} \sim \mathcal{M} w_{2}$ denotes that one can deform $w_{1}$ into $w_{2}$ along the faces of $\mathcal{M}$, as illustrated in Figure 4 . We acknowledge the evidence of this deformation as a walk homotopy between $w_{1}$ and $w_{2}$, of type $w_{1} \sim \mathcal{M} w_{2}$. The relation $(\sim \mathcal{M})$ has four constructors as follows. The first three constructors are functions to indicate that homotopy for walks is an equivalence relation, they are hrefl, hsym, and htrans. The fourth constructor, illustrated in Figure 5, is the hcollapse function that establishes the walk homotopy:

$$
\left(w_{1} \cdot \operatorname{ccw}_{\mathcal{F}}(a, b) \cdot w_{2}\right) \sim_{\mathcal{M}}\left(w_{1} \cdot \operatorname{cw}_{\mathcal{F}}(a, b) \cdot w_{2}\right),
$$

supposing one has the following,

(i) a face $\mathcal{F}$ given by $\langle A, f\rangle$ of the map $\mathcal{M}$,

(ii) a walk $w_{1}$ of type $\mathrm{W}_{U(G)}(x, f(a))$ for a node $x$ in $G$ with a node $a$ in $A$, and

(iii) a walk $w_{2}$ of type $\mathrm{W}_{U(G)}(f(b), y)$ for a node $b$ in $A$ with a node $y$ in $G$. 


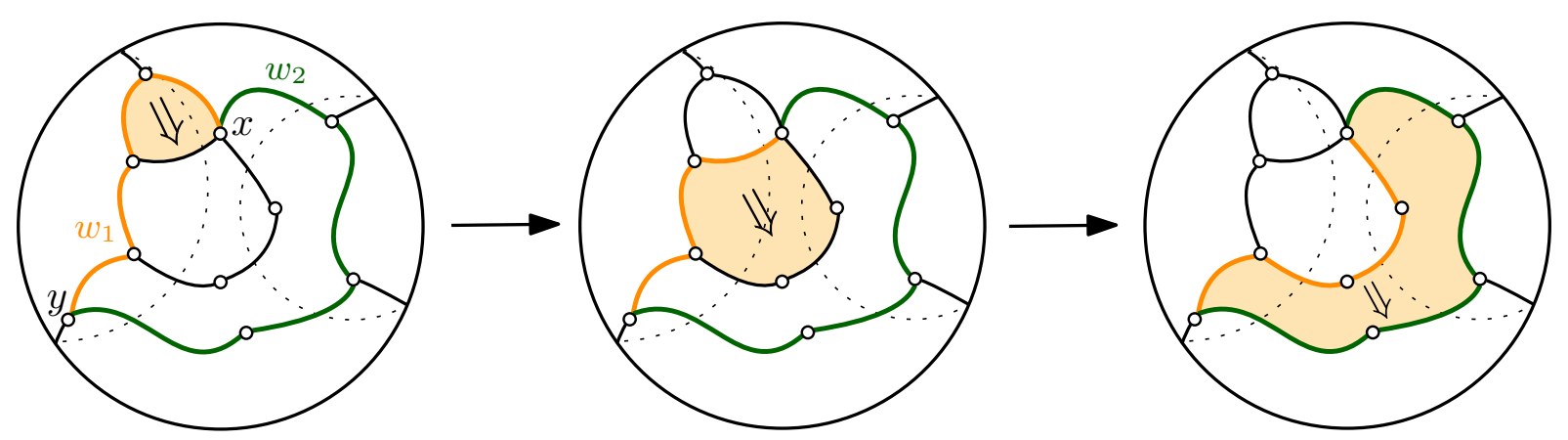

Figure 4. It is shown three homotopies between two walks from $x$ to $y$ in a graph embedded in the sphere. In each case, the arrow $(\Downarrow)$ indicates the face and the direction in which the corresponding walk deformation is performed. We obtain a homotopy between the two highlighted walks, $w_{1}$ and $w_{2}$, by composing, from left to right, the homotopies from each figure.

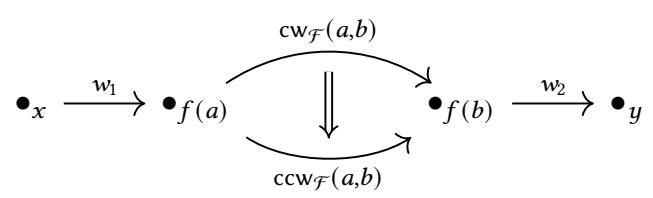

Figure 5. Given a face $\mathcal{F}$ of the map $\mathcal{M}$, we illustrate here hcollapse, one of the four constructors of the homotopy relation on walks in Definition 5.2. The arrow $(\Downarrow)$ represents a homotopy of walks.

The following lemma shows how to compose walk homotopies horizontally and vertically. We consider a map $\mathcal{M}$ for a graph $G$ and distinguishable nodes, $x, y$, and $z$ where $w$, $w_{1}$, and $w_{2}$ are walks from $x$ to $y$.

\section{Lemma 5.3.}

1. (Right whiskering) Let $w_{3}$ be a walk of type $\mathrm{W}_{U(G)}(y, z)$. If $w_{1} \sim_{\mathcal{M}} w_{2}$ then $\left(w_{1} \cdot w_{3}\right) \sim_{\mathcal{M}}\left(w_{2} \cdot w_{3}\right)$.

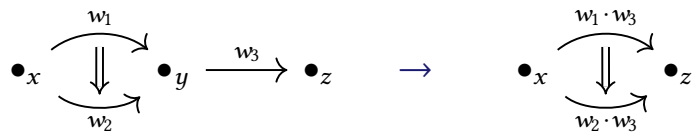

2. (Left whiskering) Let $p_{1}, p_{2}$ be walks of type $\mathrm{W}_{U(G)}(y, z)$. If $p_{1} \sim_{\mathcal{M}} p_{2}$ then $\left(w \cdot p_{1}\right) \sim_{\mathcal{M}}\left(w \cdot p_{2}\right)$.

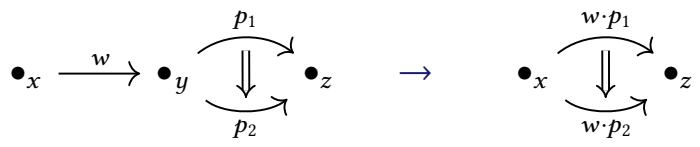

3. (Full whiskering) Let $p_{1}, p_{2}$ be walks of type $\mathrm{W}_{U(G)}(y, z)$. If $w_{1} \sim \mathcal{M} w_{2}$ and $p_{1} \sim \mathcal{M} p_{2}$, then $\left(w_{1} \cdot p_{1}\right) \sim \mathcal{M}\left(w_{2} \cdot p_{2}\right)$.

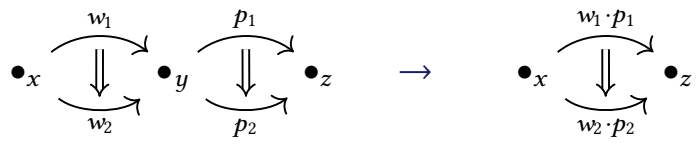

\subsection{Homotopy Walks in the Sphere}

In topology, the property of being simply connected to the sphere states that one can freely deform/contract any walk on the sphere into another whenever they share the same endpoints. This topological property of the sphere motivates the predicate in Definition 5.4, which establishes the conditions necessary for embedding a graph into a sphere. Later, we show an alternative definition for graphs with a node set in Definition 5.5. Given a distinguished face in a connected graph, being spherical for a graph embedding serves to establish elementary planarity criteria for graphs [23].

Definition 5.4. Given a graph $G$, a map $\mathcal{M}$ for $G$ is traditionally spherical if Type (5.1) is inhabited.

$$
\prod_{\left(x, y: N_{G}\right)} \prod_{\left(w_{1}, w_{2}: W_{U(G)}(x, y)\right)}\left\|w_{1} \sim_{\mathcal{M}} w_{2}\right\| .
$$

To prove a given map is spherical following Definition 5.4, one must consider the set of all possible walk-pairs for each node-pair. This is not easy, unless the set of walks follows a certain property, since the type of walks forms an infinite set. Therefore, it is proposed an alternative formulation for spherical maps based on Definition 4.29. Any walk is homotopic to its normal form, and only quasi-simple walks can be in normal form. By removing such a "redundancy" created by loops in the graph, a more convenient definition is obtained for spherical maps for graphs with discrete node set, see Definition 5.5. Furthermore, using Theorem 5.7, we show that both definitions are equivalent for graphs with discrete node set in Corollary 5.8.

Definition 5.5. Given a graph $G$, a map $\mathcal{M}$ for $G$ is spherical if the type Type (5.2) is inhabited.

$$
\prod_{\left(x, y: \mathrm{N}_{G}\left(w_{1}, w_{2}: \prod_{U(G)}(x, y)\right)\right.} \operatorname{isQuasi(w_{1})} \begin{array}{r}
\times \operatorname{isQuasi}\left(w_{2}\right) \\
\rightarrow\left\|w_{1} \sim_{\mathcal{M}} w_{2}\right\| .
\end{array}
$$

We will only refer to spherical maps as maps that follow Definition 5.5, unless stated otherwise. It is straightforward to prove that loops are homotopic to the corresponding trivial walk if a spherical map is given.

Lemma 5.6. Given a graph $G$, a spherical map $\mathcal{M}$ and $x: \mathrm{N}_{G}$, it follows that $\left\|(e \odot\langle x\rangle) \sim_{\mathcal{M}}\langle x\rangle\right\|$ for all $e: \mathrm{E}_{U(G)}(x, x)$. 
Proof. Apply $\mathcal{M}$ to the walks $(e \odot\langle x\rangle)$ and $\langle x\rangle$.

Theorem 5.7. Given a graph $G$ with a spherical map $\mathcal{M}$ and discrete set of nodes, for any walk $p: \mathrm{W}_{U(G)}(x, z)$, there exists a normal form of $p$, denoted by $\mathrm{nf}(p)$, such that $p$ is merely homotopic to $\mathrm{nf}(p)$, in the sense of Definition 5.2.

Proof. Given a walk $p$ in $U(G)$ from $x$ to $z$ of length $n$, we will construct a term of type $Q(\mathcal{M}, x, z, p)$ defined as follows.

$Q(\mathcal{M}, x, z, w): \equiv \sum_{\left(v: \mathbb{W}_{U(G)}(x, z)\right)}\left(w \rightsquigarrow{ }^{*} v\right) \times \operatorname{Normal}(v) \times\left\|w \sim_{\mathcal{M}} v\right\|$.

The proof is done by using strong induction on $n$.

- Case $n$ equals zero. The walk $p$ is the trivial walk $\langle x\rangle$, and it is then in normal form and also, by hrefl, homotopic to itself.

- Case $n$ equals one. The walk $p$ is a one-edge walk. We then ask if $x=z$.

1. If $x=z$, the walk $p$ reduces to the trivial walk $\langle x\rangle$ by $\xi_{1}$. Applying $\mathcal{M}$, one obtains evidence of a homotopy between $p$ and $\langle x\rangle$, as the two walks are quasi-simple.

2. If $x \neq z$, the one-edge walk $p$ is its own normal form and homotopic to itself by hrefl.

- Assuming that $Q\left(x^{\prime}, z^{\prime}, w\right)$ for any walk $w$ from $x^{\prime}$ to $y^{\prime}$ of length $k \leq n$, we must prove that $Q(x, z, p)$ when the length of $p$ is $n+1$.

- Therefore, let $p$ be a walk $(e \odot w)$ where $e: \mathrm{E}_{U(G)}(x, y)$ and the walk $w: \mathrm{W}_{U(G)}(y, z)$ is of length $n$. The following cases need to be considered concerning with the equality $x=y$.

1. If $x=y$ then by the induction hypothesis applied to $w$, one obtains the normal form $\operatorname{nf}(w)$ of the walk $w$, along with $r: w \rightsquigarrow \operatorname{nf}(w)$ and $h_{1}:\left\|w \sim_{\mathcal{M}} \operatorname{nf}(w)\right\|$. We ask if $x=z$ to see if $p$ is a loop.

a. If $x=z$ then the walk $p$ reduces to the trivial walk $\langle x\rangle$ by $\xi_{1}$. By applying $\mathcal{M}$ to the quasi-simple walk $\operatorname{nf}(w)$ and $\langle x\rangle, h_{2}:\|\operatorname{nf}(w) \sim \mathcal{M}\langle z\rangle\|$ is obtained. It remains to show that $p$ is homotopic to $\langle x\rangle$. Because being homotopic is a proposition, the propositional truncation in $h_{1}$ and $h_{2}$ can be eliminated to get access to the corresponding homotopies. The required walk homotopy is as follows.

$$
\begin{aligned}
p & \equiv(e \odot w) & & \\
& \sim_{\mathcal{M}} e \odot \operatorname{nf}(w) & & \left(\text { By Lemma 5.3 and } h_{1}\right) \\
& \sim_{\mathcal{M}} e \odot\langle z\rangle & & \left(\text { By Lemma 5.3 and } h_{2}\right) \\
& \sim_{\mathcal{M}}\langle x\rangle & & (\text { By Lemma 5.6 applied to } \mathcal{M}) .
\end{aligned}
$$

b. If $x \neq z$ then the walk $p$ reduces to $\mathrm{nf}(w)$ by the following calculation using $h_{1}$.

$$
\begin{aligned}
p & \equiv(e \odot w) & & \\
& \equiv(e \odot\langle x\rangle) \cdot w & & (\text { By def. of walk composition }) \\
& \rightsquigarrow^{*} w & & \left(\text { By } \xi_{3}\right) \\
& \sim^{*} \operatorname{nf}(w) & & (\text { By } r) .
\end{aligned}
$$

2. If $x \neq y$, then we split $w$ at $x$ using Lemma 4.26. Hence, two cases have to be considered: whether $x$ is in $w$ or not, see Definition 4.25 .

a. If $x \in w$, then, for every node $k$ in $G$, there are walks $w_{1}: \mathrm{W}_{U(G)}(y, k)$ and $w_{2}: \mathrm{W}_{U(G)}(k, z)$ such that $\gamma: w=w_{1} \cdot w_{2}$, along with evidence that $x \notin w_{1}$ by Lemma 4.26. By the induction hypothesis applied to $w_{1}$ and to $w_{2}$, we obtain the normal forms $\mathrm{nf}\left(w_{1}\right)$ and $\mathrm{nf}\left(w_{2}\right)$, and the terms $r_{i}: w_{i} \leadsto \operatorname{nf}\left(w_{i}\right)$ and $h_{i}:\left\|w_{i} \sim \mathcal{M} \operatorname{nf}\left(w_{i}\right)\right\|$ for $i=1,2$. The following cases concern with whether $x=z$ or not.

i. If $x=z$, the walk $p$ reduces to $\langle x\rangle$ by the rule $\xi_{1}$. To show that $p$ is homotopic to $\langle x\rangle$, let $s_{1}$ and $s_{2}$ of type, respectively, $\left\|p \sim_{\mathcal{M}} \operatorname{nf}\left(w_{2}\right)\right\|$ and $\left\|\operatorname{nf}\left(w_{2}\right) \sim \mathcal{M}\langle x\rangle\right\|$, as given below. Assuming one has the terms $s_{1}$ and $s_{2}$, by elimination of the propositional truncation and the transitivity property of walk homotopy with $s_{1}$ and $s_{2}$, the required conclusion follows. The walk homotopy $s_{1}$ is as follows.

$$
\begin{aligned}
p & \equiv(e \odot w) & & \\
& \sim_{\mathcal{M}} e \odot\left(w_{1} \cdot w_{2}\right) & & (\text { By the equality } \gamma) \\
& \sim_{\mathcal{M}}\left(e \odot w_{1}\right) \cdot w_{2} & & \text { (By assoc. property of }(\cdot)) \\
& \sim_{\mathcal{M}}\left(e \odot \operatorname{nf}\left(w_{1}\right)\right) \cdot \operatorname{nf}\left(w_{2}\right) & & \text { (By Lemma 5.3, } \left.h_{1} \text {, and } h_{2}\right) \\
& \sim_{\mathcal{M}}\langle x\rangle \cdot \operatorname{nf}\left(w_{2}\right) & & \text { (By the homotopy from } \left.h_{4}\right) \\
& \sim_{\mathcal{M}} \operatorname{nf}\left(w_{2}\right) & & \text { (By definition), }
\end{aligned}
$$

where $h_{4}:\left\|\left(e \odot \operatorname{nf}\left(w_{1}\right)\right) \sim_{\mathcal{M}}\langle x\rangle\right\|$ is given by applying the map $\mathcal{M}$ to the quasi-simple walks, $\left(e \odot \operatorname{nf}\left(w_{1}\right)\right)$ and $\langle x\rangle$. The walk $\left(e \odot \operatorname{nf}\left(w_{1}\right)\right)$ is quasi-simple by Lemma 4.10. Also, note that $x \notin \mathrm{nf}\left(w_{1}\right)$ by Lemma 4.31 and the assumption $x \notin w_{1}$. Finally, the remaining walk homotopy $s_{2}$ is obtained by applying $\mathcal{M}$ to the quasi-simple walks, $\operatorname{nf}\left(w_{2}\right)$ and the trivial walk at $x$.

ii. If $x \neq z$, then the walk $p$ reduces to $\operatorname{nf}\left(w_{2}\right)$ by the reduction reasoning in Equation (5.3). As the walk $\mathrm{nf}\left(w_{2}\right)$ is in normal form, it remains to show that $p$ is homotopic to $\operatorname{nf}\left(w_{2}\right)$. However, the reasoning is similarly to Item 2 (a)i.

$$
\begin{array}{ll}
p \equiv(e \odot w) & \\
\rightsquigarrow * e \odot\left(w_{1} \cdot w_{2}\right) & \text { (By splitting } w \text { using Lemma 4.26) } \\
\rightsquigarrow *\left(e \odot w_{1}\right) \cdot w_{2} & \text { (By assoc. property of }(\cdot)) \\
\rightsquigarrow *\langle x\rangle \cdot w_{2} & \text { (By } \left.\xi_{2} \text { applied to the loop }\left(e \odot w_{1}\right)\right) \\
\rightsquigarrow * w_{2} & \text { (By definition of walk composition) } \\
\rightsquigarrow^{*} \operatorname{nf}\left(w_{2}\right) & \text { (By the induction hypothesis). }
\end{array}
$$

b. Otherwise, there is evidence that $x \notin w$. By the induction hypothesis applied to $w$, the walk $\operatorname{nf}(w)$ is obtained, along with a reduction $r: w \rightsquigarrow \operatorname{nf}(w)$ and evidence $h:\left\|w \sim_{\mathcal{M}} \operatorname{nf}(w)\right\|$. The proof is by structural induction on the walk $\mathrm{nf}(w)$. 
i. If $\operatorname{nf}(w)$ is the trivial walk $\langle y\rangle$, then the walk $p$ reduces either to $\langle x\rangle$, if $x=z$, or to the walk $(e \odot\langle z\rangle)$, if $x \neq z$. Either way, it is possible to construct the corresponding homotopies, similarly as for Item $1 \mathrm{a}$.

ii. If the walk $\operatorname{nf}(w)$ is the composite walk $(u \odot v)$ for $u: \mathrm{E}_{U(G)}\left(y, y^{\prime}\right), v: \mathrm{W}_{U(G)}\left(y^{\prime}, z\right)$ and nodes $y^{\prime}, z: \mathrm{N}_{G}$, then we ask if $x=z$.

- If $x=z$ then the walk $p$ reduces to the trivial walk $\langle x\rangle$ by $\xi_{1}$. It remains to show that the walk $(e \odot \operatorname{nf}(w))$ is homotopic to $\langle x\rangle$. To see this, the spherical property of the map $\mathcal{M}$ is applied. Note that the walk $(e \odot \operatorname{nf}(w))$ is quasi-simple by Lemma 4.10 , as $x \notin \mathrm{nf}(w)$ by Lemma 4.31 applied to the assumption $x \notin w$.

- If $x \neq z$ then the walk $p$ reduces to the walk $(e \odot \operatorname{nf}(w))$ by $\xi_{2}$. By the propositional truncation elimination applied to the evidence of Lemma 5.3 and to the homotopy $h$, one can obtain evidence that the walk $(e \odot w)$ is homotopic to $(e \odot \operatorname{nf}(w))$. It remains to show that the composite walk $(e \odot \mathrm{nf}(w))$ is in normal form. By Lemma 4.10, this walk is quasisimple. By case analysis on the possible reductions using Definition 4.29, one proves that this walk does not reduce. Therefore, $(e \odot$ $\mathrm{nf}(w))$ is in normal form.

Corollary 5.8. The two spherical map definitions, Definition 5.4 and Definition 5.5, are equivalent when considering graphs with discrete set of nodes.

Proof. The definitions in question are propositions. Thus, it is only necessary to show that they are logically equivalent.

1. Every spherical map by Definition 5.5 is a spherical map with additional data in the sense of Definition 5.4

2. Let $\mathcal{M}$ be a spherical map by Definition 5.5 . To see $\mathcal{M}$ also satisfies Definition 5.4, let $w_{1}$ and $w_{2}$ be two quasi-simple walks from $x$ to $y$. We must now exhibit evidence that $w_{1}$ is homotopic to $w_{2}$. By Theorem 5.7, a walk homotopy $h_{1}$ between $w_{1}$ and the normal form $\operatorname{nf}\left(w_{1}\right)$ exists. Similarly, one can obtain a term $h_{2}$ of type $\left\|w_{2} \sim \mathcal{M} \operatorname{nf}\left(w_{2}\right)\right\|$.

$$
\begin{aligned}
w_{1} & \sim \mathcal{M} \operatorname{nf}\left(w_{1}\right) & & \left(\text { By } h_{1}\right. \text { from Theorem 5.7) } \\
& \sim \mathcal{M} \operatorname{nf}\left(w_{2}\right) & & \left(\text { By } h_{3}\right. \text { from Definition 5.5) } \\
& \sim_{\mathcal{M}} w_{2} & & \left(\text { By } h_{2}\right. \text { from Theorem 5.7). }
\end{aligned}
$$

On the other hand, recall that walks in normal form are quasi-simple walks by definition. Therefore, it is possible to get $h_{3}:\left\|\mathrm{nf}\left(w_{1}\right) \sim \mathcal{M} \operatorname{nf}\left(w_{2}\right)\right\|$ by applying the spherical property of the map $\mathcal{M}$ to $\operatorname{nf}\left(w_{1}\right)$ and $\mathrm{nf}\left(w_{2}\right)$. By the elimination of the propositional truncation applied to $h_{1}, h_{2}$, and $h_{3}$, the required evidence of a homotopy between $w_{1}$ and $w_{2}$ can be obtained, as stated in Equation (5.4).

\section{Related Work}

In other areas of mathematics unrelated to type theory, considering homotopy for graph-theoretical concepts, for example, is not new. There are several proposals of the concept of homotopy for graphs using a few discrete categorical constructions [9]. Many of these constructions use the $x$-homotopy notion, defined as a relation based on the categorical product of graphs in the Cartesian closed category of undirected graphs. Since a walk of length $n$ in a graph $G$ is simply a morphism between a path graph $P_{n}$ into $G$, the notion of homotopy for walks is there defined as homotopy between graph homomorphisms. The looped path graph $I_{n}$ is used to define the homotopy of these morphisms-in a manner similar to the interval $[0,1]$ for the concept of homotopy between functions in homotopy theory. As a source of more results, it is possible to endow the category of undirected graphs with a 2-category structure by considering homotopies of walks as 2-cells, as described by Chih and Scull [3].

On the reduction relation on walks and spherical maps, this work is related to polygraphs used in the context of higher-dimensional rewriting systems. Recent works by Kraus and von Raumer $[13,14]$ use ideas in graph theory, higher categories, and abstract rewriting systems to approximate a series of open problems in HoTT. In the same vein, the internalisation of rewriting systems and the implementation of polygraphs in Coq by Lucas $[15,16]$ was found to be related to Kraus and von Raumer's approach. One fundamental object in the work by the authors mentioned above is that of an $n$-polygraph, also called computad.

An $n$-polygraph is a (higher dimensional) structure that can serve, for example, to analyse reducing terms to normal forms and comparing reduction sequences on abstract term rewriting systems. The following is a possible correspondence to relate these ideas within the context of our work. The notion of a 1-polygraph [14, §2]-which is given by two sets $\Sigma_{0}$ and $\Sigma_{1}$, and two functions $s_{0}, t_{0}: \Sigma_{1} \rightarrow \Sigma_{0}$-is equivalent to the type of graphs in Definition 3.1. An object is a node, a reduction step is an edge, and a reduction sequence $a \rightsquigarrow *^{*} b$ is a walk between nodes $a$ to $b$. A (closed) zig-zag is a (cycle) walk in the symmetrisation of the graph representing the reduction relation. A (generalised) 2-polygraph [14, Def. 25] consists of a type $A$, a set of reduction steps on $A$, and all rewriting steps between zig-zags. Then, the notion of 2-polygraph on $A$ will correspond to a graph $G$ representing the type $A$ with the set of all walks in $G$ and the collection of walk homotopies in the symmetrisation $U(G)$ for a given combinatorial map.

Using the previous interpretation for polygraphs, one may state that a graph with a spherical map holds properties such as terminating, closed under congruence, cancels inverses, and it has a Winkler-Buchberger structure [14, Eq. 32-35]. The related concept of homotopy basis of a 2-polygraph [14, 
Def. 28] may be seen as the set obtained from Definition 5.4 without using propositional truncation in the corresponding type.

On the other hand, Noetherian induction for closed zigzags $[14, \S 3.5]$ addresses a similar issue we investigated herein. In this work, we found out that to prove certain properties, as the normalisation theorem in Theorem 5.7 for graphs with a spherical map and a discrete set, it was only necessary to consider (cycle) walks without inner loops. One can prove other properties related to walk homotopies for graphs with spherical maps, not only considering the property on a cycle walk but any walk. This approach relies on the machinery of quasi-simple walks in Section 4.3 and the loop reduction relation on walks in Section 4.6. Our loopreduction relation is likely locally confluent [14, § 3.3], but without uniqueness of normal forms. We leave the proof of these properties as future work because they were not required here. We will also investigate in-depth the extent to which the constructions given by Kraus and von Raumer, as well as by Lucas, are not only related but applicable to our main project of graph theory in HoTT [21].

Finally, on the computer formalisation side, the use of formal systems to formalise graph-theoretical results on the computer is not a novelty. The proof of the four-colour theorem (FCT) in Coq by Gonthier [8] is one famous example that works with hypermaps-a similar notion to combinatorial maps, as defined in Definition 5.1. However, both the type theory and the goal of the constructions are substantially different from our exposition. There are other relevant projects in the field and extensive libraries of graph theory in Coq [6], Isabelle/HOL [20], and Lean [11]. However, to the best of our knowledge, few efforts use a proof-relevant dependent type theory like HoTT and a proof assistant like Agda. We find only the work mentioned earlier by Kraus and von Raumer $[13,14]$ to be related to our Agda development; their work contains a formalisation of their results in a version of the proof assistant Lean compatible with HoTT.

In other formal developments like the HoTT Coq Library [2], the UniMath Library [28], and the Standard Library of Cubical Agda, only the basic definitions are available (e.g. the type of graphs, graph homomorphisms, and diagrams). Future work might involve porting our development into one of these libraries.

\section{Concluding Remarks}

This work proves some non-trivial results for directed multigraphs using a proof-relevant approach in the language of homotopy type theory. This work supports an ongoing project to define planarity criteria and other concepts of graph theory in HoTT [22, 23] formalised in Agda [21].

In our formalisation, each definition and theorem presented herein is related to a term in the proof assistant Agda. This approach was helpful to reveal and confirm that only a subset of HoTT was necessary to perform all the proofs in this development. Precisely, we only need the intensional Martin-Löf type theory equipped with universes, function extensionality, and propositional truncation. No other higher inductive type is required. It is worth noting that without considering propositional truncation, it would not have been possible to define our main theorems. The propositional truncation allows us to model the mere existence of an object in theory correctly.

This work's primary contributions are Theorem 5.7, and especially Corollary 5.8. In summation, Theorem 5.7 states that we can normalize any walk to a normal form that is walk-homotopic to it whenever the graph has a discrete node set and is embedded in the sphere. On the other hand, Corollary 5.8 establishes an equivalence between two definitions of embeddings in the sphere for graphs with a discrete node set. Except for this last result, the machinery shown in this paper was utterly unexpected and developed solely to find evidence for our initial conjecture. For characterising embeddings of finite graphs in the sphere, one needs to consider only the finite set of walks without internal loops. Using the results given herein, one can devise a (brute-force) algorithm to determine whether an embedding is spherical or not. Future work will be devoted to implementing this algorithm. To the best of our knowledge, we provided the minimum to demonstrate Theorems 4.37 and 5.7 and Corollary 5.8.

\section{Acknowledgments}

The author thanks Håkon R. Gylterud for very helpful discussions on various issues related to this paper. Thanks to Marc Bezem and the anonymous reviewers for the comments, references and suggestions that improved this document. Thanks to the Department of Informatics at the University of Bergen for funding this research. Last but not least, thanks to the Agda developer team for providing and maintaining the proof assistant used to check the results of this work.

\section{References}

[1] Steve Awodey. 2012. Type Theory and Homotopy. In Epistemology versus Ontology. Springer Netherlands, Pitt, Usa, 183-201. https: //doi.org/10.1007/978-94-007-4435-6_9

[2] Andrej Bauer, Jason Gross, Peter LeFanu Lumsdaine, Michael Shulman, Matthieu Sozeau, and Bas Spitters. 2017. The HoTT Library: A Formalization of Homotopy Type Theory in Coq. In Proceedings of the 6th ACM SIGPLAN Conference on Certified Programs and Proofs (Paris, France) (Cpp 2017). Association for Computing Machinery, New York, NY, USA, 164-172. https://doi.org/10.1145/3018610.3018615

[3] Tien Chih and Laura Scull. 2020. A homotopy category for graphs. Journal of Algebraic Combinatorics (2020). https://doi.org/10.1007/ s10801-020-00960-5

[4] Jesper Cockx, Dominique Devriese, and Frank Piessens. 2016. Eliminating dependent pattern matching without K. Journal of Functional Programming 26 (2016), e16. https://doi.org/10.1017/s0956796816000174

[5] Reinhard Diestel. 2012. Graph Theory, 4th Edition. Graduate texts in mathematics, Vol. 173. Springer, Hamburg, Germany. https://doi.org/ 10.1007/978-3-662-53622-3 
[6] Christian Doczkal and Damien Pous. 2020. Graph Theory in Coq: Minors, Treewidth, and Isomorphisms. F. Autom. Reason. 64, 5 (2020), 795-825. https://doi.org/10.1007/s10817-020-09543-2

[7] Martín Hötzel Escardó. 2019. Introduction to Univalent Foundations of Mathematics with Agda. CoRR abs/1911.00580 (2019). arXiv:1911.00580 http://arxiv.org/abs/1911.00580

[8] Georges Gonthier. 2008. Formal proof-the four-color theorem. Notices of the AMS 55, 11 (2008), 1382-1393. https://doi.org/10.1.1.141.714

[9] Alexander Grigor'yan, Yong Lin, Yuri Muranov, and Shing-Tung Yau. 2014. Homotopy theory for digraphs. http://arxiv.org/abs/1407.0234

[10] Jonathan L Gross and Thomas W Tucker. 1987. Topology Graph Theory. Dover, Ny, Usa. 387 pages.

[11] Alena Gusakov, Bhavik Mehta, and Kyle A. Miller. 2021. Formalizing Hall's Marriage Theorem in Lean. arXiv:2101.00127 [math.CO]

[12] Wen Kokke, Jeremy G. Siek, and Philip Wadler. 2020. Programming language foundations in Agda. Sci. Comput. Program. 194 (2020), 102440. https://doi.org/10.1016/j.scico.2020.102440

[13] Nicolai Kraus and Jakob von Raumer. 2020. Coherence via WellFoundedness. In Proceedings of the 35th Annual ACM/IEEE Symposium on Logic in Computer Science. Acm. https://doi.org/10.1145/3373718. 3394800

[14] Nicolai Kraus and Jakob von Raumer. 2021. A Rewriting Coherence Theorem with Applications in Homotopy Type Theory. arXiv:2107.01594 [cs.LO]

[15] Maxime Lucas. 2019. An implementation of polygraphs. (2019). https: //hal.archives-ouvertes.fr/hal-02385110 working paper or preprint.

[16] Maxime Lucas. 2020. Abstract rewriting internalized. arXiv:2002.06004 [math.CT]

[17] Conor McBride. [n.d.]. A polynomial testing principle. https:// personal.cis.strath.ac.uk/conor.mcbride/PolyTest.pdf

[18] Bengt Nordström. 1988. Terminating general recursion. Bit 28, 3 (Sept. 1988), 605-619. https://doi.org/10.1007/bf01941137
[19] Ulf Norrell. [n.d.]. Towards a practical programming language based on dependent type theory. $\mathrm{PhD}$ thesis. Chalmers University of Technology. https://research.chalmers.se/en/publication/46311

[20] Lars Noschinski. 2015. Formalizing Graph Theory and Planarity Certificates. Ph.D. Dissertation. Technischen Universität München, Germany. https://d-nb.info/1104933624/34

[21] Jonathan Prieto-Cubides. 2019. Investigations on graph-theoretical constructions in Homotopy type theory - Agda formalisation. https: //doi.org/10.5281/zenodo.5775569 Work-in-progress.

[22] Jonathan Prieto-Cubides and Håkon Robbstand Gylterud. 2019. Planar graphs in HoTT. 25th International Conference on Types for Proofs and Programs, TYPES (2019). http://www.ii.uib.no/ bezem/abstracts/ TYPES\%5F2019\%5Fpaper\%5F37

[23] Jonathan Prieto-Cubides and Håkon Robbestad Gylterud. 2022. On planarity of graphs in homotopy type theory.

(2022). arXiv:1601.05035 [cs.LO] https://arxiv.org/abs/

[24] W. T. Tutte. 1960. Convex Representations of Graphs. Proceedings of the London Mathematical Society s3-10, 1 (1960), 304-320. https: //doi.org/10.1112/plms/s3-10.1.304

[25] W. T. Tutte. 1963. How to Draw a Graph. Proceedings of the London Mathematical Society s3-13, 1 (1963), 743-767. https://doi.org/10.1112/ plms/s3-13.1.743

[26] The Univalent Foundations Program. 2013. Homotopy Type Theory: Univalent Foundations of Mathematics. https://homotopytypetheory. org/book, Institute for Advanced Study.

[27] Vladimir Voevodsky. 2010. The equivalence axiom and univalent models of type theory. (Talk at CMU on February 4, 2010). , 1-11 pages. https://arxiv.org/abs/1402.5556

[28] Vladimir Voevodsky, Benedikt Ahrens, Daniel Grayson, et al. [n.d.]. UniMath - a computer-checked library of univalent mathematics. available at https://unimath.org. https://github.com/UniMath/ UniMath 